\title{
Hydrodynamic memory can boost enormously driven nonlinear diffusion and transport
}

\author{
Igor Goychuk ${ }^{1}$, and Thorsten Pöschel ${ }^{1}$ \\ ${ }^{1}$ Institute for Multiscale Simulation, Friedrich-Alexander University \\ of Erlangen-Nueremberg, Cauerstr. 3, 91058 Erlangen, Germany
}

(Dated: July 3, 2020)

\begin{abstract}
Hydrodynamic memory force or Basset force is known since the 19th-century. Its influence on Brownian motion remains, however, mostly unexplored. Here, we investigate its role in nonlinear transport and diffusion within a paradigmatic model of tilted washboard potential. In this model, a giant enhancement of driven diffusion over its potential-free limit [Phys. Rev. Lett. 87, 010602 (2001)] presents a well-established paradoxical phenomenon. In the overdamped limit, it occurs at a critical tilt of vanishing potential barriers. However, for weak damping, it takes place surprisingly at another critical tilt, where the potential barriers are clearly expressed. Recently we showed [Phys. Rev. Lett. 123, 180603 (2019)] that Basset force could make such a diffusion enhancement enormously large. In this paper, we discover that even for moderately strong damping, where the overdamped theory works very well when the memory effects are negligible, substantial hydrodynamic memory unexpectedly makes a strong impact. First, the diffusion boost occurs at non-vanishing potential barriers and can be orders of magnitude larger. Second, transient anomalous diffusion regimes emerge over many time decades and potential periods. Third, particles' mobility can also be dramatically enhanced, and a long transient super-transport regime emerges.
\end{abstract}

\section{INTRODUCTION}

Non-Markovian hydrodynamic memory effects due to Boussinesq-Basset force emerging in the motion of macroscopic bodies with fluctuating velocity are known since the 19th-century [1-3] and still present an active field of research [4, 5]. Their influence on Brownian motion [6 9] remains, however, largely unexplored, even though they are known to effect famous algebraic tail in the velocity autocorrelation function (VACF) of Brownian particles [10, 11], which was found first in molecular dynamic simulations by Alder and Wainwright 12]. Neither diffusion, nor transport are, however, affected asymptotically in the absence of nonlinear force fields, even if a transient superdiffusion is engendered [13]. The existence and importance of such memory effects was experimentally manifested for free diffusion of colloidal particles [14], and, more recently, for particles trapped in parabolic potentials [15-17]. It raises the question of their general role and importance in nonlinear transport and diffusion [18], where the model of tilted washboard potentials serves as a paradigm in condensed matter physics and far beyond [7 9]. Within this model, a giant enhancement of driven diffusion [19 21] over its potential-free limit is a wellestablished paradoxical phenomenon in the overdamped limit [8, 22, 23], where the inertial effects are entirely negligible. It occurs at a critical potential tilt of vanishing potential barriers [8, 20, 21] with applied constant force $f_{c}^{(1)}=1$ in the units used in this paper. Inertial effects in nonlinear diffusion beyond thermal equilibrium are less studied [7, 8] and brought many surprises [24 28] lately. However, the influence of hydrodynamic memory

\footnotetext{
* igor.goychuk@fau.de, corresponding author
}

effects on such a nonlinear driven diffusion and transport came only recently in the spotlight of attention [18]. For weak damping, a giant enhancement of diffusion occurs at another critical tilt, where the potential barriers are clearly expressed [26-28]. Then, profound memory effects make such an enhancement enormously large and result in a substantial prolongation of a transient superdiffusion regime [18].

To understand the mechanism of a resonancelike enhancement of diffusion in the corresponding weaklydamped regime, the picture of motion bistability originally developed by Risken and collaborators [7, 29] in the absence of hydrodynamic memory becomes crucially important. There exists a critical friction value $\gamma_{0}^{(c)} \approx 1.193$ [7] (in scaled units used below) such that for a smaller friction at zero temperature there emerges a friction-dependent critical tilt value $f_{c}^{(3)}$ (our notations are different from used in [7]) such that for tilting forces between two critical values, $f_{c}^{(3)}<f<f_{c}^{(1)}$ the motion is bistable at zero temperature with thermal fluctuations neglected. An excellent account of this bistability is given in [30] in the context of stochastic resonance problem. Depending on the starting point in the phase space, any particle will either end in one of potential wells due to frictional losses, or run indefinitely, when losses are compensated by the energy delivered from the external field. In this bistable regime and at a finite temperature $T$, velocity of particle exhibits bistable fluctuations for a sufficiently small friction $\gamma_{0} \ll \gamma_{0}^{(c)}$. They can be modeled and well understood as thermally activated fluctuations in a bistable velocity pseudo-potential $V(v)=-k_{B} T \ln [P(v)]$, where $P(v)$ is velocity distribution and $k_{B}$ is Boltzmann constant [27, 28, 31]. One potential minimum corresponds to $v_{1}=0$ (trapped particles), and another one to $v_{2}=f / \gamma_{0}$ (running particles, 
units are scaled). Near minima, $P(v)$ is approximately Gaussian (Maxwell distribution) with a thermal velocity width 27, 31]. The giant enhancement of diffusion occurs at the condition of equal pseudo-potential minima or equal probabilities [27, 28] for the particle to be trapped or run in an intermittent Lévy walk like fashion [24, 32 34]. Such a critical condition can also be obtained in a generic model of velocity-bistable active diffusion [35]. For underdamped dynamics in washboard potential, the diffusion maximum occurs at some $f_{c}^{(2)}$, $f_{c}^{(3)}<f_{c}^{(2)}<f_{c}^{(1)}$ satisfying this condition.

Upon taking hydrodynamic memory influence for a small $\gamma_{0}$ into account, this basic picture remains approximately valid upon some essential modifications [18]. First, the diffusion enhancement becomes strongly amplified and sharpened (suppressed outside of the narrow maximum region). Second, the distribution of particle velocities in the running state (near its maximum) is broader than Maxwellian. This effect can be characterized by either enhanced kinetic temperature in the running state, or by a smaller effective mass of the particle in this state. The latter interpretation is preferred because it is convenient to characterize the whole velocity distribution by a kinetic temperature measured by its width [18, 26, 36 38]. Particles become kinetically hot in the bistable regime. Third, an effective friction experienced by particles becomes enlarged by the hydrodynamic memory friction. This leads to an effective suppression of the asymptotically normal transport in comparison with the memoryless case. However, transient regime of looking anomalously fast transport, $\langle\delta x(t)\rangle \sim t^{\kappa_{t}}$, with $\kappa_{t}>1$ can be prolonged enormously. Likewise, transient superdiffusion, $\left\langle\delta x^{2}(t)\right\rangle \sim t^{\kappa_{d}}$, with $\kappa_{d}>1$ can also be drastically prolonged in time. It is due to the changed kinetics of the transitions between two macrostates of velocity Lévy walk, which becomes anomalously slow, stretched-exponential, instead of exponential in the memoryless case - the fourth profound feature introduced by hydrodynamic memory. Furthermore, like in the case of asymptotically superdiffusive transport [37 39], long hyperdiffusive regimes, $\kappa_{d}>2$, are present due to transiently growing in time kinetic temperature. In the memoryless case, such regimes are also present [26]. However, they are much shorter.

Now, novel profound questions emerge: How these interesting features introduced by hydrodynamic memory are modified beyond the $\gamma_{0} \ll \gamma_{0}^{(c)}$ regime studied in [18]? Is hydrodynamic memory still important for $\gamma_{0}$ equal and even larger than Risken's $\gamma_{0}^{(c)}$, when dynamics becomes overdamped? This question is very important because Brownian motion in fluids is typically overdamped. For example, in experimental works [15, 17] colloidal particles are overdamped and, nevertheless, exhibit resonances caused by the hydrodynamic memory. Next, does hydrodynamic memory always increase an effective friction or it can also make that smaller, e.g. for a sufficiently large $\gamma_{0}$, and how large is large? For ex- ample, some results in recent Ref. [40] for transport in a critically tilted piecewise linear periodic potential at $T=0$ imply that this can be the case. Next, remains picture of bistable velocity fluctuations valid for sufficiently large $\gamma_{0}$, which is yet smaller than $\gamma_{0}^{(c)}$ ? Actually, some results presented in the Supplemental Material [41] of Ref. [18] imply "no" already for $\gamma_{0}$ larger than about 0.25 , which was confirmed in a recent detailed study [42]. This feature means that the running velocity state is not necessarily monostable unless $\gamma_{0}$ is small enough. The numerical simulations reveal that already for $\gamma_{0}=0.3$, the velocity distribution can be trimodal, see Fig. 7, (a) in [41], and, especially, the panel (c) therein, for $\gamma_{0}=0.7$, where the running state consists, in fact, of two velocity substates with $P(v)$ maxima at $v_{2}^{(1)}$ and $v_{2}^{(2)}$ such that $v_{2}^{(1)}<v_{2}=f / \gamma_{0}<v_{2}^{(2)}$. Moreover, $v_{2}$ corresponds to the minimum (!) and not maximum of $P(v)$, as bistable picture of $V(v)$ [26, 27], valid for sufficiently small $\gamma_{0}$ only [18, 42], can misleadingly imply. For a critical tilt $f=1$ in Fig. 7, (d) in [41], the minimum at $v=0$ (trapped state) disappears, and the running state remains bistable. It means that velocity fluctuations can remain bistable even for an overcritical tilt, when the trapped states are absent. Hence, the case of nearly overdamped dynamics is not trivial, even if to neglect crucial memory effects.

Below we show that even for a moderately strong damping, within a seemingly overdamped regime, hydrodynamic memory unexpectedly makes a very profound impact. As a general implication, it means that hydrodynamic memory effects, whose neglect might earlier seem intuitively be well justified, can nevertheless profoundly affect nonlinear transport and diffusion. They should not be generally ad hoc neglected in further research. Theory of nonlinear Brownian motion in fluids should be rethought and revisited from this angle of view.

\section{MODEL AND THEORY}

We consider one-dimensional transport and diffusion of spherical Brownian particles with radius $R$ and mass $m=4 \pi \rho R^{3} / 3$ ( $\rho$ is the particles' mass density) in a fluid with kinematic viscosity $\mu$ and density $\rho_{f}$ governed by a fractional Langevin equation (FLE) [8, 13, 15, 38, 43, 44]

$$
\begin{array}{r}
m^{*} \ddot{x}(t)+\eta_{0} \dot{x}(t)+\eta_{\alpha-\infty} \hat{D}_{t}^{1 / 2} \dot{x}(t) \\
=f(x)+\xi_{0}(t)+\xi_{\alpha}(t)
\end{array}
$$

in a periodic force-field

$$
f(x)=f_{c}^{(1)} \sin \left(x / x_{0}\right)+f
$$

with amplitude $f_{c}^{(1)}=U_{0} / x_{0}$. Here, $U_{0}$ is the amplitude of the corresponding washboard potential with period $L=2 \pi x_{0}$, which is biased by a constant driving force $f$. Trapped states exist only below the critical value $f_{c}^{(1)}, f<f_{c}^{(1)}$. In Eq. (1), $m^{*}=m+$ 
$2 \pi \rho_{f} R^{3} / 3$ is a fluid-renormalized mass of a Brownian particle [3, 11, 45], $\eta_{0}=6 \pi R \rho_{f} \mu$ is Stokes viscous fric-

tion, ${ }_{-\infty} \hat{D}_{t}^{\alpha-1} v(t):=\frac{1}{\Gamma(2-\alpha)} \frac{d}{d t} \int_{-\infty}^{t} d t^{\prime} v\left(t^{\prime}\right) /\left(t-t^{\prime}\right)^{\alpha-1}$, with $\alpha=3 / 2$, is Riemann-Liouville fractional derivative [13, 46], and $\eta_{\alpha}=\eta_{0} \sqrt{\tau_{r}}$ is a fractional friction coefficient. The corresponding memory term in the FLE reflects hydrodynamic memory, which is characterized by a relaxation time scale $\tau_{r}=R^{2} / \mu$ entering $\eta_{\alpha}$. It presents the Boussinesq-Basset force, which is derived within similar approximations as the Stokes friction, however, for a particle with fluctuating velocity (non-steady Stokes flow) [1-3]. Roughly speaking, $\tau_{r}$ is a characteristic time for a backflow induced by the body motion to diffuse over its size. Inertial effects in the particle's dynamics are also not always negligible. The characteristic velocity relaxation time, $\tau_{v}=m^{*} / \eta_{0}$ (obtained in neglecting the memory effects), is $\tau_{v}=\tau_{r}\left(2 \rho / \rho_{f}+1\right) / 9$, in terms of $\tau_{r}$ and the ratio $\rho / \rho_{f}$ of the body and fluid densities. For example, in the case of a neutrally buoyant particle, $\rho_{f}=\rho, \tau_{v}=\tau_{r} / 3$, and $\tau_{v}=\tau_{r}$ at $\rho=4 \rho_{f}$. It means that unless the Brownian particle is very heavy with respect to fluid, hydrodynamic memory is not neglible once the particle's inertia becomes important, especially given a slow algebraic character of this memory decay. We recast the Boussinesq-Basset force in the form of memory friction [13, 18, 38, 44], $\int_{-\infty}^{t} \eta\left(t-t^{\prime}\right) \dot{x}\left(t^{\prime}\right) d t^{\prime}$, with a singular memory kernel $\eta(t)$ corresponding to the operator of the Riemann-Liouville fractional derivative. For $1<\alpha<2, \eta(t>0)=-\eta_{\alpha} t^{-\alpha} /|\Gamma(1-\alpha)|<0$, however, $\int_{0}^{t} \eta\left(t^{\prime}\right) d t^{\prime} \sim t^{1-\alpha}>0$ is always positive and tends to zero with $t \rightarrow \infty$. This term is absent for $v=\dot{x}=$ const. However, it is always present in the realm of Brownian particles, where it must be complemented by the corresponding unbiased thermal Gaussian force $\xi_{\alpha}(t)$ obeying the fluctuation-dissipation relation (FDR) [11, 47], $\left\langle\xi_{\alpha}\left(t^{\prime}\right) \xi_{\alpha}(t)\right\rangle=k_{B} T \eta\left(\left|t-t^{\prime}\right|\right)$, which follows from the fundamental fluctuation-dissipation theorem (FDT) [11, 47]. $\xi_{\alpha}(t)$ provides a naturally emerging instance of the fractional Gaussian noise or fGn [48]. By the same token, $\left\langle\xi_{0}\left(t^{\prime}\right) \xi_{0}(t)\right\rangle=2 k_{B} T \eta_{0} \delta\left(\left|t-t^{\prime}\right|\right)$, as in the standard Langevin equation, where $\xi_{0}(t)$ is a white Gaussian noise, which like fGn is a singular stochastic process with infinite variance existing only in a class of distributions. FLE (1) presents an important example of general nonlinear Generalized Langevin Equation or GLE [11, 44, 47].

Periodic potentials acting on Brownian microparticles can be created by a lattice of optical vortices [22] or optical tweezers [23] (with $L$ in the sub-micron range), or, e.g., by nanoimprint lithography [49], for nanoparticles (down to nanometer scale). The FLE description was confirmed experimentally for colloidal particles in parabolic traps [15 17], where hydrodynamic effects were measurable and even caused resonances in the case of almost overdamped dynamics [15]. They, however, never were studied for nonlinear Brownian transport and diffusion until recently [18], even theoretically, except for a model case, where the Stokes friction was ad hoc neglected [38]. Indeed, in the case of potentialfree diffusion, $U_{0}=0$, the memory effects do not affect the diffusion coefficient, $D_{0}=k_{B} T / \eta_{0}$, asymptotically. However, they do cause some relatively short transient superdiffusion [13] and profoundly modify the stationary VACF, $\langle v(t) v(0)\rangle_{\text {st }}$. Namely, it universally acquires asymptotically a long algebraic tail, $\langle v(t) v(0)\rangle_{\mathrm{st}} \sim$ $v_{T}^{2} \sqrt{\tau_{r} / \pi} /\left(2 \gamma_{0} t^{3 / 2}\right)$ [10, 13, 18], where $v_{T}=\sqrt{k_{B} T / m^{*}}$ is thermal velocity and $\gamma_{0}=\eta_{0} / \mathrm{m}^{*}$. This tail has first been found in molecular-dynamics simulations by Alder and Wainwright [12]. Moreover, the initial decay of VACF is stretched-exponential and not exponential, in the case of strong yet realistic memory effects [18]. The case of driven nonlinear diffusion is capable of further surprises [18].

We shall scale distance in $x_{0}$, time in $\tau_{0}=x_{0} \sqrt{m^{*} / U_{0}}$, which is inverse circular frequency of oscillations at the bottom of potential wells in the absence of friction and bias, energy in $U_{0}$, and temperature as $\tilde{T}=k_{B} T / U_{0}$. In these units, $f_{c}^{(1)}=1$ and dimensionless $\tilde{\gamma}_{0}=\gamma_{0} \tau_{0}$ (tilde will be mostly omitted in the following) measures the strength of normal friction. For $\eta_{\alpha}=0$, the unbiased intrawell dynamics is overdamped for $\gamma_{0} \geq 2$. Furthermore, $\gamma_{\alpha}=\eta_{\alpha} / m^{*}$ in these units reads $\gamma_{\alpha}=$ $3 \sqrt{\gamma_{0} /\left(1+2 \rho / \rho_{f}\right)}$, which is maximal, $\gamma_{\alpha}^{(\max )}=3 \sqrt{\gamma_{0}}$, in the limit of ultralight particles, $\rho / \rho_{f} \rightarrow 0$. The memory effects are fully negligible in the opposite limit $\rho / \rho_{f} \rightarrow \infty$, and are expected to be strong for $\rho \sim \rho_{f}$ or smaller. FLE does not allow for analytical solutions for the considered nonlinear dynamics and we solved it numerically [18], as detailed in the Appendix A.

\section{RESULTS AND DISCUSSION}

\section{A. Influence of memory effects on bistability phase diagram}

As mentioned in Introduction, the fundamental feature of driven underdamped dynamics in tilted washboard potential in the absence of memory effects is its bistability [7. Namely, for $\gamma_{0}$ less than a critical value $\gamma_{0}^{(c)} \approx 1.193$ [7], there exists a critical force $f_{c}^{(3)}<f_{c}^{(1)}$ such that for $f<f_{c}^{(3)}$, any trajectory in the phase space will end eventually at $T=0$ in one of the potential wells. However, for $f_{c}^{(3)} \leq f<f_{c}^{(1)}$, the deterministic running solutions emerge and co-exist with the trapped ones, whereas for $f>f_{c}^{(1)}$, only the running solutions remain. This critical force value depends on $\gamma_{0}$. For a small friction, $f_{c}^{(3)} \approx 4 \gamma_{0} / \pi$, see Fig. 11.26 in Ref. [7] and red doubledash-dotted line in Fig. 1, (a) of this paper. Numerical $f_{c}^{(3)}\left(\gamma_{0}\right)$ is shown by full black line with diamond symbols in this figure. It increases monotonously with $\gamma_{0}$ and $f^{(3)}=f^{(1)}$ at $\gamma_{0}=\gamma_{0}^{(c)}$.

How does hydrodynamic memory affect the Risken's phase diagram? We answer first this important question. 

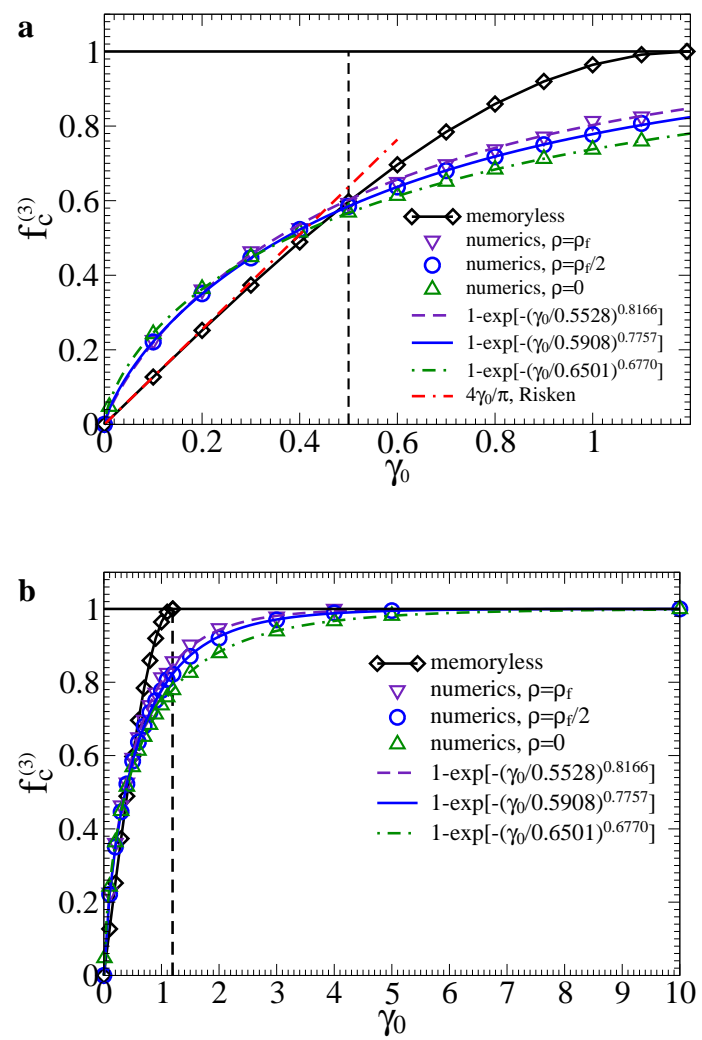

FIG. 1. Phase diagram of bistability at $T=0$. Dependence of the critical force $f_{c}^{(3)}$ on $\gamma_{0}$ without and in the presence of hydrodynamic memory effects for three fixed values of ratio $\rho / \rho_{f}$ shown in the plot. For $f<f_{c}^{(3)}$, there are no running states. Every trajectory eventually ends in a potential well (trapped solutions). For $f_{c}^{(3)}<f<f_{c}^{(1)}=1$, running trajectories co-exist with trapping solutions, and for $f>1$ the only running solutions remain. In the memoryless case [7], $f_{c}^{(3)} \approx 4 \gamma_{0} / \pi$, for $\gamma_{0} \lesssim 0.25$, see red double-dash-dotted line in panel (a). For for $\gamma_{0} \lesssim 0.5$, memory effects increase the effective friction, which can be judged upon the correspondingly increased $f_{c}^{(3)}$. This trend is changed to the opposite for $\gamma_{0}>0.5$. The vertical line in panel (a) at $\gamma_{0}=0.5$ helps to realize this. Moreover, the regime of bistability extends far beyond the Risken's $\gamma_{0}^{(c)} \approx 1.193$ [see vertical line in panel (b)], as panel (b) manifests.

Profound memory effects for $\rho=\rho_{f}$ (neutrally buoyant condition) and $\rho=\rho_{f} / 2$ (a relatively lighter than fluid particle) used mostly in the numerical simulations below profoundly change the phase diagram in Fig. 1 where also the limiting case of $\rho \rightarrow 0$ (maximal memory effects) is depicted. In all three cases, numerical data are well approximated by a stretched-exponential dependence $f_{c}^{(3)} \approx 1-\exp \left[-\left(\gamma_{0} / b\right)^{a}\right]$, with $0<a<1$, and $b>0$ shown in the plot by dashed indigo line $\left(\rho=\rho_{f}\right)$, full blue line $\left(\rho=\rho_{f} / 2\right)$, and dash-dotted green line $(\rho \rightarrow 0)$, which come through the corresponding different symbols depicting the numerical results. For $\gamma_{0} \ll b$ this yields $f_{c}^{(3)} \propto \gamma_{0}^{a}$ instead of $f_{c}^{(3)} \propto \gamma_{0}$ in the memoryless case. It presents the first important result of this paper. This

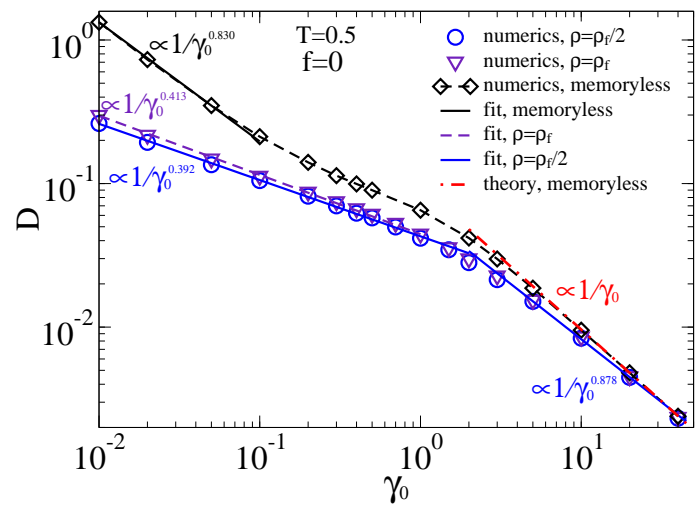

FIG. 2. Dependence of nonlinear diffusion coefficient on viscous friction in the case of unbiased diffusion at $T=0.5$. Two values of the ratio of particles to fluid density are considered, $\rho / \rho_{f}=1$ and $\rho / \rho_{f}=1 / 2$, as well as the memoryless case, for comparison, see major text for detail. Numerical data are shown by indigo triangles for $\rho=\rho_{f}$ (with a small- $\gamma_{0}$ fit depicted by dashed indigo line coming through the symbols), blue circles for $\rho=\rho_{f} / 2$ (with small and large $\gamma_{0}$ fits depicted by full blue lines coming through the symbols), and dashed black line with diamonds, in the case of memoryless dynamics. Analytical result for a large-friction limit of memoryless dynamics is depicted by dash-dotted red line, and a small- $\gamma_{0}$ fit to this dynamics by full black line.

dramatic change means: (1) For $\gamma_{0}$ smaller than about $\gamma_{0}=0.5$ shown by the vertical line in panel (a) of Fig. 1. ever-increasing with lowering $\rho / \rho_{f}$ memory effects make an effective friction larger. However, for $\gamma_{0} \gtrsim 0.5$, the opposite tendency is seen in panel (a). In particular, even for $\gamma_{0}$ essentially larger than $\gamma_{0}^{(c)}$, the bistability region extends dramatically, cf. panel (b). It means that even for $\gamma_{0}>\gamma_{0}^{(c)}$ the memory correlations can induce running solutions at $T=0$ and $f_{c}^{(3)}<f<f_{c}^{(1)}$, where, otherwise, all Brownian particles would remain asymptotically trapped forever. Then, the memory makes an effective friction smaller. This result agrees with the conclusions in Ref. 40] that hydrodynamic memory can induce transport at $T=0$ in a situation where it would be absent otherwise, which was obtained therein for a critically tilted piecewise linear potential. In the studied case, pertinent tilts can be essentially smaller than the critical value $f_{c}^{(1)}$. One should emphasize that we are dealing here with a strongly driven transport. It is the second result of paramount importance, which provides a key for understanding our numerical results below.

\section{B. Influence of memory on normal diffusion in unbiased potential}

Next, we expect that the dependence of the normal diffusion coefficient on $\gamma_{0}$ will also be dramatically changed in a periodic potential, and we will check this hypothesis. Indeed, in the case of unbiased diffusion, it is well-known 

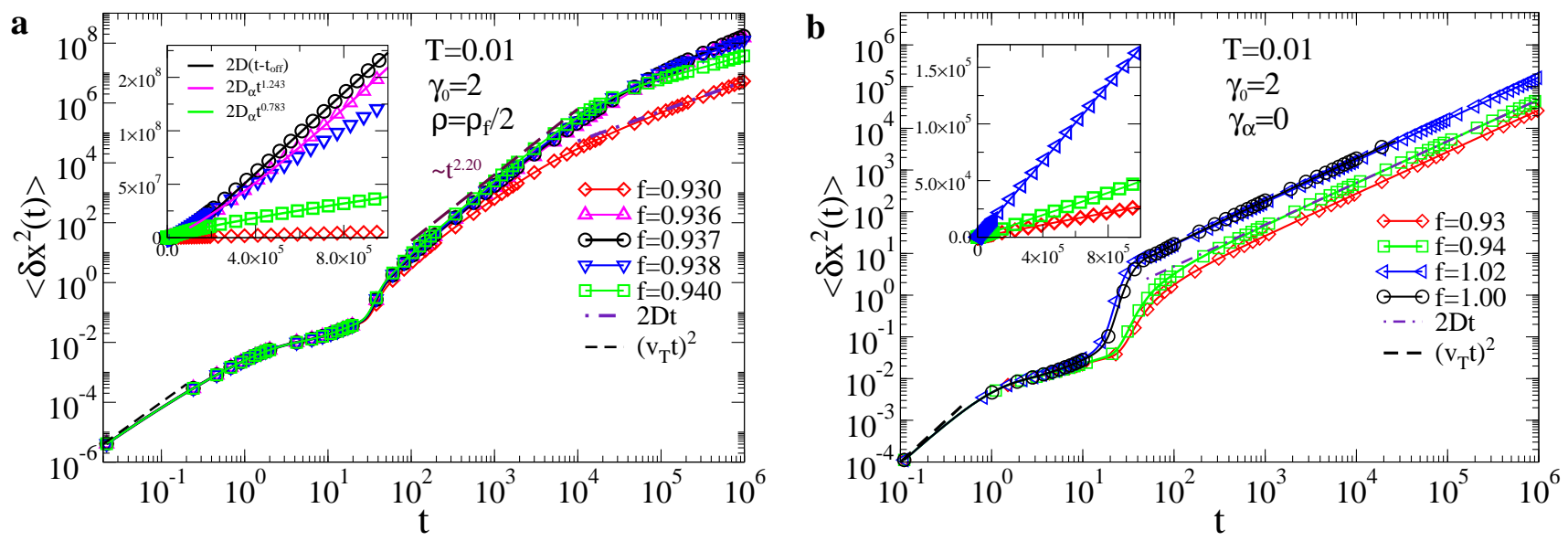

FIG. 3. Dependence of the particles position variance on time for (a) non-Markovian and (b) Markovian diffusion at $T=0.01$, $\gamma_{0}=2$ and several values of force shown in the plot. In the non-Markovian case $\gamma_{\alpha}=3$, which corresponds to $\rho=\rho_{f} / 2$. $M=10^{5}$ particles are used in the ensemble averaging. Particles are always initially localized at $x=0$ within one potential well with their velocities Maxwell-distributed. Initial diffusional spread is always briefly ballistic $(t \rightarrow 0)$, as indicated by the corresponding dashed black lines in main plots. Insets show the same dependencies in linear plot. Panel (b) makes clear that without memory effects a normal diffusion regime is quickly established once particles leave the potential well. However, memory effects in panel (a) introduce a very long intermediate hyperdiffusive regime. Moreover, diffusion is still anomalously fast at the end of simulations for $f$ close to the critical tilt $f_{c}^{(2)} \approx 0.937$, see full black line with circles in (a), including inset, which corresponds to resonance-like value of $D / D_{0}$ in Fig. 4 (a), and also full magenta line with triangles up for $f=0.936$ in (a). It is fitted by a superdiffusion dependence in the inset of (a). The case of $f=0.937$ in this inset we fit with $2 D\left(t-t_{\text {off }}\right)$ in the last half time decade of simulations to derive $D$ from numerics, see the main text. For $f=0.940$ a very long subdiffusive regime astoundingly emerges in the last two time decades of simulations, cf. the light green line with squares in (a), including inset. Full lines with different symbols correspond to different values of $f$ shown in the main plots, except for the inset in (a), where three lines correspond to the fits shown in this inset.

that for strong friction, $\gamma_{0} \gg 1, D \propto 1 / \gamma_{0}$, and for the considered potential simple and well-known LifsonJackson result $D=D_{0} / I_{0}^{2}\left(U_{0} / k_{B} T\right)$ holds [7, 9, [50]. Here, $I_{0}(x)$ is a modified Bessel function. This inverse friction proportionality holds at any $k_{B} T / U_{0}$. Less known is that this scaling, $D \propto 1 / \gamma_{0}$, is valid also for weak friction, $\gamma_{0} \ll 1$, however, in the limit of large barriers $U_{0} \gg k_{B} T$ only [7]. In the memoryless case, our numerics (depicted by black dashed line with diamonds) perfectly agree with the Lifson-Jackson result for $\gamma_{0} \geq 1$, see dash-dotted red line in Fig. 2. Hydrodynamic memory, however, remarkable changes this result even for strong friction in the range $2<\gamma_{0}<40$. Namely, instead of the inverse friction dependence, our numerics are more consistent with $D \propto 1 / \gamma_{0}^{0.878}$, even for rather strong friction in the mentioned range, see full blue line with circles and triangles in this figure. For much larger friction, the Lifson-Jackson result remains, however, valid, even in the limiting case $\rho \rightarrow 0$. Furthermore, for a small friction $\gamma_{0}<1, D \propto 1 / \gamma_{0}^{a}$ in Fig. 2. with $a \approx 0.413$ for $\rho=\rho_{f}$ (dashed indigo line coming through triangles), and $a \approx 0.392$ for $\rho=\rho_{f} / 2$ (full blue line coming through circles), which is very different from $a \approx 0.83$ in the case of normal diffusion (full black line coming through diamonds). The latter one deviates from the high-barrier theoretical value $a=1$ because $k_{B} T / U_{0}$ is not small enough [7], only 0.5 . The discovered non-trivial dependencies of $D$ on $\gamma_{0}$ due to the memory effects present the third important result of this work. It should be mentioned also that in the case of such equilibrium unbiased diffusion hydrodynamic memory always increases an effective friction because it makes the diffusion coefficient in Fig. 2 smaller at all $\gamma_{0}$.

\section{Enormous boost of diffusion acceleration due to the memory effects}

Furthermore, we study an enormous acceleration of diffusion due to the memory effects in the onset of an overdamped regime, $\gamma_{0}=2$, where the memoryless diffusion enhancement is already nicely described by the results of the overdamped theory, cf. Eq. (8) in Ref. [20], see the corresponding comparison in our Fig. 目 (a). It is one of the greatest surprises of this work, which extends and complements recent findings in Ref. [18]. For $\gamma_{0}=2$ and $\rho=\rho_{f} / 2, f_{c}^{(3)} \approx 0.920$ in Fig. 1. The giant enhancement of diffusion is hence to expect for some critical value $f_{c}^{(2)}$ such that $f_{c}^{(3)}<f_{c}^{(2)}<f_{c}^{(1)}$. For a small $\gamma_{0}$, this $f_{c}^{(2)}$ corresponds to the case, where the probabilities of trapped and running states become roughly equal [18, 27, [28]. At odds with intuition based on our earlier results for $\gamma_{0}=0.1$ in Ref. [18], this resonance-like enhancement occurs for $\gamma_{0}$ essentially exceeding $\gamma_{0}^{(c)}$ of the Markovian case! Moreover, now it does not correspond to the situa- 

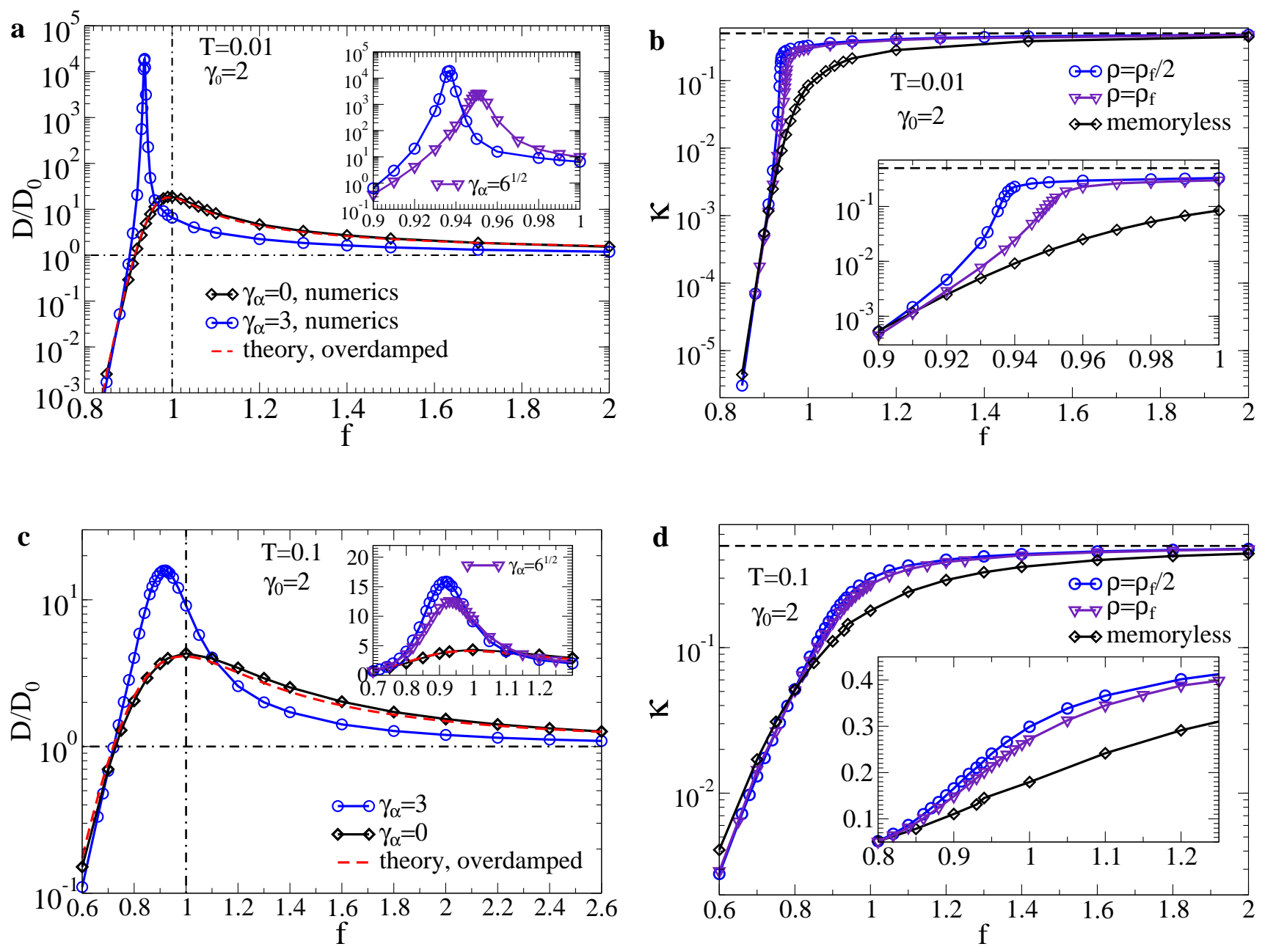

FIG. 4. Diffusion coefficient and nonlinear mobility. (a,c) Enhancement of driven diffusion over the free-diffusion limit depending on the applied force $f$ for (a) $T=0.01$ and (c) $T=0.1, \gamma_{0}=2$ and three values of $\gamma_{\alpha}$ shown in the plot. $\gamma_{\alpha}=3$ corresponds to $\rho=\rho_{f} / 2$. The inset resolves a sharp peak for this value in the main plot for a shorter range of $f$ and depicts for comparison the results for $\gamma_{\alpha}=\sqrt{6}$, which correspond to $\rho=\rho_{f}$. The red dashed line in the main plots depicts the analytical result of overdamped theory given by Eq. (8) in Ref. [20]. It remarkably agrees with the memoryless result of simulations, which includes, however, the inertial effects completely. (b,d) Nonlinear mobility $\kappa v s$. driving force $f$ for $(b) T=0.01$ and $(\mathrm{d}$ ) $T=0.1, \gamma_{0}=2$ and two values of $\gamma_{\alpha}$ corresponding to $\rho=\rho_{f} / 2$ and $\rho=\rho_{f}$, as well as for the memoryless case. The inset helps to resolve dramatic changes around the corresponding critical values $f_{c}^{(2)}$, where the increase of mobility due to the memory effects is dramatic. Both far below and far above $f_{c}^{(2)}$ the memory-induced effects in $\kappa$ are negligible. For $f=2$, the linear mobility regime of $\kappa_{0}=1 / \eta_{0}$ is already almost achieved.

tion of equal probabilities of trapped and running states, see below. The physics of this enhancement is hence different. Some of the results on non-Markovian diffusion for $\gamma_{0}=2, \rho=\rho_{f} / 2$ and $T=0.01$ are shown in Fig. 3. (a). Let us to compare them with the results on the matching Markovian diffusion in Fig. 3. (b). First of all, in the Markovian case the normal diffusion regime is already well-established on the scale exceeding the lattice period $2 \pi,\left\langle\delta x^{2}(t)\right\rangle>4 \pi^{2}$, for all values of $f$ in this plot. Quite on the contrary, a very long regime of transient superdiffusion emerges in panel (a), which extends on huge many potential periods, when $f$ becomes close to the resonance-like value $f_{c}^{(2)} \approx 0.937$ (for $\rho=\rho_{f} / 2$ ), see in Fig. 4 (a). To derive the results for the asymptotic value $D / D_{0}$ from the numerical data in Fig. 3, we fit the $\left\langle\delta x^{2}(t)\right\rangle$ dependence by $2 D\left(t-t_{\text {off }}\right)$, where $t_{\text {off }}$ is some offset time required to account for a very long transient period of anomalous diffusion. It can be neglected only

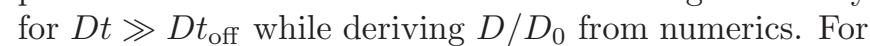
example, for a sub-resonance value $f=0.93<f_{c}^{(2)}$ in Fig. 3. (a), the asymptotic normal diffusion regime is already well established. However, for the resonance value $f=f_{c}^{(2)}=0.937$, it is not, see results depicted with circles in the inset of Fig. 3, (a), and compare with the case $f=0.936$ (triangles up) to realize why one needs $t_{\text {off }}$. The corresponding value of $D / D_{0}$ in Fig. 4. (a) 
is an estimate from below. The fantastic thousandfold enhancement $D / D_{0}$ by about 18944 times at peak, over the result in neglect of the memory effects, which enhancement factor is about 18.2 times "only", is, in fact, even larger. However, we cannot quantify it better because the proper normal diffusion limit is not reachable in simulations. With diminishing memory effects along with increasing $\rho / \rho_{f}$, this enhancement weakens, see inset in Fig. 4. (a), for neutrally buoyant case $\rho=\rho_{f}$ with $\gamma_{\alpha}=\sqrt{6}$ at $\gamma_{0}=2$, and, nevertheless, it is still impressively strong. In this case, see inset in Fig. [4 (a), $f_{c}^{(2)} \approx 0.951$ with $D / D_{0} \approx 2590$ at maximum. Generally, with diminishing $\gamma_{\alpha}$ at fixed $\gamma_{0}>\gamma_{0}^{(c)}, f_{c}^{(2)}$ moves towards $f_{c}^{(1)}$ and $D$ diminishes gradually to its memoryless value.

Next, at $f=0.936$ and $f=0.937$ in Fig. 3, (a), diffusion is anomalously fast, even near to the end simulation. Inset therein makes this clear for $f=0.936$, where $\left\langle\delta x^{2}(t)\right\rangle \propto t^{\kappa_{d}}$ with $\kappa_{d}=1.243$, which is a fit alternative to $2 D\left(t-t_{\text {off }}\right)$ dependence that is not shown for this $f$ value. It is a striking result: Even for $\gamma_{0}=2$, hydrodynamic memory effects can turn normal diffusion into superdiffusion over many time decades corresponding to thousands of lattice periods! This long-lasting transient superdiffusion is explained by kinetic heating, as Fig. 6 of Appendix $\mathrm{C}$ makes clear. Indeed, the kinetic temperature $T_{k}(t)=m^{*}\left\langle\delta v^{2}(t)\right\rangle / k_{B}[26$, 36 38] defined by the the variance of the velocity distribution $P(v)$ increases dramatically, i.e., Brownian particles become kinetically hot, with their $T_{k}$ substantially exceeding $T$ of the surrounding fluid. Velocity distribution in Appendix $\mathrm{C}$ is also not Maxwellian, bimodal in the regime of enhanced diffusion. However, differently from the case of small $\gamma_{0}$ [18, 27, 28], for the considered large $\gamma_{0}, f_{c}^{(2)}$ does not correspond, even approximately, to the situation where the local maximum of $P(v)$, which corresponds to the running state, compares in amplitude with the local maximum corresponding to the trapped states. Moreover, for $f=f_{c}^{(2)}>f_{c}^{(3)}$, there are actually two running substates (within a two-state approximation), as $P(v)$ in Fig. 7 reveals. Its first local maximum does not correspond to $v=0$. Moreover, the integral $\int_{-\infty}^{v_{c}} P(v) d v$, where $v_{c}$ is the velocity value, which separates two running substates, indicates that nearly $90 \%$ of all particles belong to the first running substate. It is very different from the case of low friction. In the latter case, the peak of $D / D_{0}$ does correspond to the situation, where, roughly speaking, one-half of the particles are temporarily trapped, while another one-half run [27, 28], also in the presence of memory effects [18]. Hence, for a sufficiently large $\gamma_{0}$, the mechanism of a huge enhancement due to memory effects differs from one established for small $\gamma_{0}$ [18]. Nevertheless, for strong memory effects, $f_{c}^{(2)}$ seems to still roughly correspond to the maximum of stationary non-equilibrium $T_{k}^{(\text {st) }}$ vs. $f$, like for a small $\gamma_{0}$ [18], as we detail in Appendix C.

It is very different from the matching case, where the memory effects are neglected. Indeed, for the considered $\gamma_{0}$ and in the memoryless case, the maximum of $D$ has nothing in common with the maximum of $T_{k}^{(\text {st) }}$, cf. Appendix C. The particles can become kinetically very hot also in the absence of memory effects at $\gamma_{0}=2$, see in Fig. [6 (b). It seems to be first in contradiction with the fact that the overdamped theory remarkably well describes the numerical results on $D / D_{0}$ enhancement in our Fig. 4. (a). We defer a detailed explanation of this puzzle somewhere else. In short, due to inertial effects, there emerge very fast oscillations in the non-equilibrium but stationary VACF for $f>1$, even for sufficiently large $\gamma_{0} \sim 2-10$. Following Green-Kubo relation [11], diffusion coefficient is integral of VACF and those fast oscillations reduce $D$ in spite of still growing (for $f>1$, in the memoryless case) $T_{k}^{\text {(st) }}$. It resolves the discussed apparent contradiction. However, it leads to a paradox: Becoming ever hotter particles (for a certain intermediate interval of overcritical forces $f>1$ ) diffuse ever slower, and $D / D_{0}$ drops for $f>1$. This very striking non-equilibrium phenomenon emerging due to inertial effects in nonlinear memoryless driven dynamics for a sufficiently large, but not too large friction $\gamma_{0}$ was entirely overlooked thus far. It will be studied in detail somewhere else. The inertial effects in the Brownian motion are highly nontrivial and remain insufficiently studied until now, even in the simplest paradigmatic systems like one considered.

Next, quite embarrassing, for $f=0.94$, a very prolong hyperdiffusive, faster than ballistic, regime (see the main plot in Fig. 3, a) changes in the last time decade into a subdiffusive regime with $\left\langle\delta x^{2}(t)\right\rangle \propto t^{0.783}$ (see light green line with squares in inset therein). The latter one is, for sure, transient. Nevertheless, its appearance for an already saturated with time $T_{\mathrm{k}}^{\text {(st) }}$, which exceeds $T$ by more than four and a half times, see in Fig. 6 is physically really puzzling. Mathematically, it, of course, just corresponds to a very long transition from superlinear $\left\langle\delta x^{2}(t)\right\rangle \propto t^{2.2}$ scaling with time to an asymptotically linear one. Quite paradoxically, we are dealing here with a hot subdiffusion. For a small $\gamma_{0}$, such a regime occurs due to a transient cooling after the maximum of $T_{k}(t)$ in time is passed [18]. It was revealed also for a periodically driven memoryless underdamped diffusion in a ratchet potential [51]. In the present case, the underlying mechanism is, however, different. Notice also that in this case $t_{\mathrm{off}}$ is negative and $2 D\left(t-t_{\mathrm{off}}\right)$ fit overestimates $D$. With a further increase of $f$, the asymptotically normal regime is gradually established.

\section{Diffusion at higher temperature}

With the increase of temperature $T$, the influence of memory effects on the diffusion enhancement becomes smaller. Nevertheless, for $T=0.1$ in Fig. 4. (c) it is still manifestly present. First, the diffusion maximum occurs at some $f_{c}^{(2)}<1$. Second, at its maximum the 
enhancement is three to four times stronger than in the memoryless case, that also is pretty well described by the analytical result of the overdamped theory. Indeed, for $\rho=\rho_{f} / 2$ at in Fig. 目 (c), $f_{c}^{(2)}=0.92$. Notice that it equals $f_{c}^{(3)}$ in this case and $D / D_{0} \approx 15.87$ at maximum. It should be compared with the maximum $D / D_{0} \approx 4.32$ at $f=1$ in the memoryless case. The memory-induced boost is about by 3.67 times. For $\rho=\rho_{f}$ therein, $f_{c}^{(2)} \approx$ 0.93 with maximal $D / D_{0} \approx 12.57$. The increase is still impressive nearly 2.91 times. The effect is not small.

\section{Hydrodynamic memory boosts nonlinear mobility in a subcritical tilt region}

Finally, we study the influence of memory effects on the particles' nonlinear mobility $\kappa(f)=v(f) / f$, where $v$ is the mean particles velocity $v=\langle\delta x(t)\rangle / t$ defined at the last half time decade of simulations by using a $v\left(t-t_{\text {off }}\right)$ fit to numerical $\langle\delta x(t)\rangle$. The mean displacement of particles is shown in Fig. 5. (a), for the case with strong memory, $\rho=\rho_{f} / 2, T=0.01, \gamma_{0}=2$ and several values of force in a narrow interval, $f=0.93-0.94$, around $f_{c}^{(2)}=0.937$. It should be compared with Fig. [5. (b), where the matching memoryless case, $\gamma_{\alpha}=0$, is depicted. First of all, it is worth noting that in the limit $t \rightarrow 0$, the initial transport is universally ballistic, $\langle\delta x(t)\rangle \sim f t^{2} / 2\left(m^{*}=1\right)$. For a very brief initial time period, the periodic potential does not matter. The particles are prepared at $x=0$, which is not a mechanically equilibrium state in the biased case, and they move first accelerating towards nearest potential minimum. Then, they start to equilibrate and $\langle\delta x(t)\rangle$ temporary saturates. Notice also that the range of $f$ variation in this figure is so small that all the curves practically coincide during the equilibration process with the potential well. Next, the particles start to escape out of the potential well and travel over many potential periods being driven by $f$; become occasionally trapped in other potential wells during this process and rereleased (notice that $f$ is rather close to $f_{c}^{(1)}=1$ from below). In the memoryless case, transport is practically normal, $\langle\delta x(t)\rangle \propto v\left(t-t_{\text {off }}\right.$ ) (where $t_{\mathrm{off}}$ is some offset time) once $\langle\delta x(t)\rangle$ exceeds $2 \pi$ - the potential period. Also, in the case with memory, but for subcritical $f=0.930$ shown by red line with diamonds in panel (a), the normal transport regime is established relatively fast. However, for $f$ closer to $f_{c}^{(2)}$, a dramatic enhancement of transport occurs. Notice that then transport becomes very sensitive to tiny $f$ variations (compare with the initial regime for $t<100$ !). Remarkably, a very long transient supertransport regime $\langle\delta x(t)\rangle \propto t^{\kappa_{t}}$ with $\kappa_{t} \approx 1.43$ emerges, which can cover about one thousand potential periods. Of course, even in this regime, the transport is slower in absolute terms than in the absence of hindering periodic potential. However, hydrodynamic memory greatly accelerates transport in the periodic potential near-to-critically tilted, i.e., the memory effects in synergy with thermal fluctuations greatly help to overcome the residual potential bumps on the way. Interesting, even at the end of simulations (which take several days being run with double numerical precision and $M=10^{5}$ particles in parallel on high-performance professional GPU processors, for one curve presented) the transport remains anomalously fast, $\kappa_{t} \approx 1.1$, in this case, see inset of panel (a) for more detail. The fit with $v\left(t-t_{\text {off }}\right)$, see, e.g., in the discussed inset for $f=0.940$ then still underestimates the actual value of $v$. In Fig. 4, (b) of the main text, we provided the nonlinear mobility based on such an estimate in the pertinent cases, where the normal transport regime was not possible to reach in numerics.

The results for mobility are depicted at $T=0.01$ in Fig. 4. (b) for three values of $\gamma_{\alpha}$, including the memoryless case. For $f$ smaller than $f=0.9<f_{c}^{(3)}$, the influence of memory is negligible in this plot. Likewise, for a very large $f$, the regime of linear mobility in the absence of potential, $\kappa_{0}=1 / \eta_{0}=0.5$, is gradually achieved already for $f \geq 2$. However, near to $f_{c}^{(2)}$ the enhancement of mobility by the memory effects is tremendous. For $\rho=\rho_{f} / 2$, at $f=0.94, \kappa=0.2288$, whereas without memory effects it is merely 0.009156 . The boost of mobility is about 25 times! This is a very striking effect. Likewise, for $\rho=\rho_{f}$, at $f=0.96, \kappa=0.2283$, whereas without memory effects it is merely 0.02524 . The memory-caused increase is by impressive nearly 9 times.

Important to mention is that for $T=0.01$ and $f$ close to $f_{c}^{(2)}$, the transport is, in fact, anomalously fast during the major period of simulations, as Fig. 5. (a) reveals. First, $\langle\delta x(t)\rangle \propto t^{1.43}$ for intermediate times after particles started to leave the potential well and move in the force direction. This super-transport regime lasts for $t$ until about $2 \times 10^{4}$. During this time, particles move over about a thousand potential periods. Notice that the transport power-law exponent $\kappa_{t}=1.43$ is close to 1.5, which would correspond to the case of Stokes friction contribution ad hoc neglected [38]. One can state that the corresponding super-transport regime is manifested here in the presence of Stokes friction, which is an important result. Second, even at the end of the simulation, the corresponding power-law exponent still did not relax to unity being about 1.1. The corresponding estimates of the mean velocity, like one shown in the inset of Fig. 5. (a) for $f=0.94$ underestimate, in fact, the corresponding value of $v$ and the mobility in Fig. 4. (b). Comparison with Fig. [5 (b), where such a regime is absent, makes clear that this supertransport emerges due to hydrodynamic memory effects.

\section{Transport at higher temperature}

Finally, we provide the readers with the results on nonlinear mobility enhancement for a larger $T=0.1$ or smaller $U_{0} /\left(k_{B} T\right)=10$ in Fig. 目. (d). The memory- 

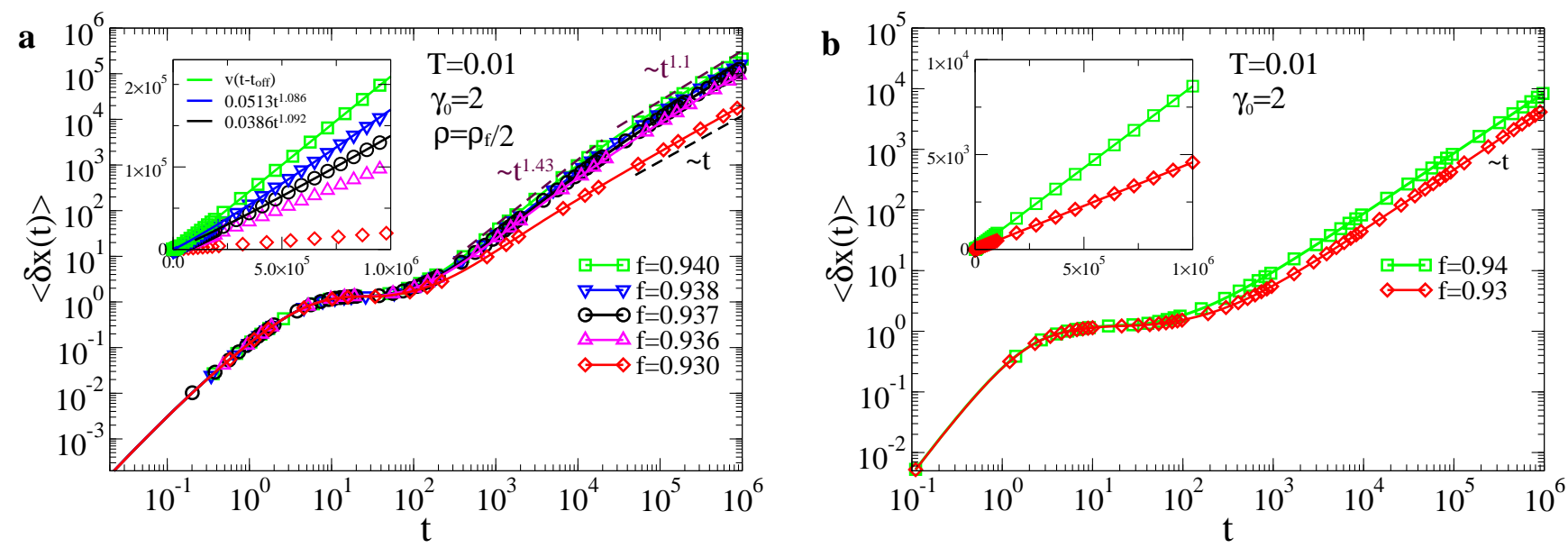

FIG. 5. Dependence of the particles displacement on time in (a) non-Markovian and (b) Markovian transport at $T=0.01$, $\gamma_{0}=2$ and several values of force shown in the plot. In panel (a), $\gamma_{\alpha}=3$, which corresponds to $\rho=\rho_{f} / 2 . M=10^{5}$ particles are used in the ensemble averaging. Particles are always initially localized at $x=0$ within one potential well with their velocities Maxwell-distributed. Insets show the same dependencies in linear plot. Panel (b) makes clear that without memory effects a normal transport regime is quickly established once particles leave the potential well. However, memory effects in panel (a) introduce a very long intermediate supertransport regime. Moreover, transport is still anomalously fast at the end of simulations for $f$ close to the critical tilt $f_{c}^{(2)} \approx 0.937$, see inset, which corresponds to resonance-like value of $D / D_{0}$ in Fig. 4 , (a) of the main text. Full lines with different symbols correspond to matching values of $f$ shown in the main plots, except for the inset in (a), where three lines correspond to the fits shown in this inset.

induced increase of mobility becomes less impressive, and, nevertheless, it remains still significant. For example, in this figure, at $f=0.93, \kappa=0.2106$ for $\rho=\rho_{f} / 2$, $\kappa=0.1882$ for $\rho=\rho_{f}$, and $\kappa=0.1310$ in the memoryless case. The enhancement is by $60.75 \%$ and $43.66 \%$ with respect to the memoryless case, correspondingly. It is not small at all.

We conclude that also at higher temperatures the memory effects can significantly boost both diffusion and transport in near-to-critically tilted periodic potentials over the case, where such effects are neglected. Hydrodynamic memory can also suppress diffusion outside the resonance-like critical enhancement regime, see, e.g., in Fig. 4. (a) for $f>1$ and in Fig. 4. (c) for $f>1.15$ and $f<0.7$. Similar suppression was earlier discussed in Ref. [18] for a weak friction case of $\gamma_{0}=0.1$. Transport is generally enhanced for $f>f_{c}^{(3)}$, however, it can also be suppressed for $f<f_{c}^{(3)}$, see, e.g., in Fig. 目 (d) for $f<0.8$. In this respect, the case of moderately strong $\gamma_{0}=2$ considered here is different from the case of small $\gamma_{0}=0.1$ investigated in Ref. 18. Our Fig. 10xplains why. In any case, influence of hydrodynamic memory on strongly nonequilibrium stochastic transport can be very significant even for moderately strong friction in the case of sufficiently light particles.

\section{SUMMARY AND CONCLUSIONS}

In summary, in this paper, we showed within a paradigmatic model of driven nonlinear Brownian transport and diffusion that hydrodynamic memory effects primarily neglected thus far in the theory of nonlinear Brownian motion could profoundly influence both diffusion and transport even for a relatively strong Stokes friction. First, enormous resonance-like enhancement of diffusion can occur for a potential tilt, which is subcritical for such a Stokes friction taken alone, where the pertinent overdamped theory [20] describes already very well the numerical results in the negligence of such memory effects. This memory-induced surplus enhancement can be giant for light particles, by several orders of magnitude, depending on temperature and the ratio $\rho / \rho_{f}$ which measures strength of the memory effects. Second, also transport can be enhanced enormously at the corresponding resonance-like tilt. Third, the transient superdiffusive and supertranport regimes can last for a long time while covering thousands of lattice periods. Particles can become kinetically very hot in these anomalous regimes, with their kinetic temperature well above the temperature of the surrounding liquid. Fourth, even undriven thermally equilibrium diffusion in periodic potentials exhibit novel features manifested by an inverse fractional dependence of the diffusion coefficient on the Stokes friction strength.

The experimental verification of these intriguing and highly surprising effects can be expected, cf. Appendix $\mathrm{B}$, in least viscous liquids like liquid helium at $T=4$ $\mathrm{K}$ (above $\lambda$ point, still a normal fluid), for hollow microparticles with tailored $\rho$, which are trapped in optically created potentials [22], or even in more viscous yet more common fluids like diethyl ether for nanoparticles in nanoimprinted periodic potentials created by meth- 
ods of lithography [49]. In such micro- and nanofluidic systems, the inertial effects in Brownian motion can become essential being greatly amplified by the hydrodynamic memory effect, as this work showed. We expect that it will attract the interest of not only theoreticians but also experimental scientists and spark subsequent research work.

\section{ACKNOWLEDGMENT}

We acknowledge support by the Regional Computer Centre Erlangen, Leibniz Supercomputing Centre of the Bavarian Academy of Sciences and Humanities, as well as University of Potsdam (Germany), which kindly provided GPU high-performance computational facilities for doing this work. This research was funded by the Deutsche Forschungsgemeinschaft (German Research Foundation), Grant GO 2052/3-2.

\section{Appendix A: Numerical approach}

Numerical integration of FLE (10) is based on an approximation of the power-law scaling part of memory kernel by a sum of exponentials (a Prony series expansion) and hyper-dimensional Markovian embedding of underlying non-Markovian dynamics [18, 38, 44, 52]. The method works very well and leads to results, which often practically coincide within the numerical precision tolerance of $5-6 \%$ (can be made better) with the analytical results available in case of linear dynamics. The memory kernel approximation reads [18, 38]

$$
\eta(t)=\sum_{i=1}^{N} \eta_{i}\left[2 \delta(t)-\nu_{i} \exp \left(-\nu_{i} t\right)\right],
$$

where $\eta_{i}=k_{i} / \nu_{i}, \quad k_{i}=C_{\alpha}(b) \eta_{\alpha} \nu_{i}^{\alpha} /|\Gamma(1-\alpha)|$, and $\nu_{i}=\nu_{0} / b^{i-1}$. The sum of exponentials obeys a fractal scaling with a scaling parameter $b$. It approximates the power-law decay [44, 52 54] of this memory kernel, so that $\int_{0}^{\infty} \eta(t) d t=0$. The choice of $\nu_{0}$ is related to the time step of simulation $\Delta t$, which was $\Delta t=0.002$ in most simulations. To avoid numerical instability, $\nu_{0} \Delta t$ should be smaller than one.

The power-law regime extends in this approximation from a short time (high-frequency) cutoff, $\nu_{0}^{-1}$, to a large time (small frequency) cutoff, $\tau_{h}=\tau_{l} b^{N-1}$. The choice of $N$ is dictated by the maximal time $t_{\max }$ of simulations: $\tau_{h}$ should exceed $t_{\max }$ by at least several times. The accuracy of the approximation between two cutoffs is controlled by the scaling parameter $b>1$. The smaller $b$, the better the accuracy. However, a larger $N$ is then required. With $b=5$ and $C_{\alpha}(b)=1.78167$ [18, 38] it is about $6 \%$ for $t$ between 0.05 and $10^{6}$ for $\nu_{0}=100$ and $N=13$. It can be slightly improved to $5 \%$ by choosing $C_{\alpha}(b)=1.816$, which is used in most simulations in this paper. When required, the discussed accuracy can drastically be improved to about $0.003 \%$ between 0.07 and $10^{6}$ for $\nu_{0}=100$ and $N=38$ with $b=2$ and $C_{\alpha}(b)=0.782134$. This choice would, however, also essentially increase the simulation time because of a much larger embedding dimension. Since we are interested in reaching a maximal time range in computer simulations, we use the same embedding with $b=5$ and $N=13$ as earlier [18, 38]. Even in this case, simulations are very time-consuming. It takes several days to reach $t_{\text {max }}=10^{6}$ on professional GPU processors (double precision accuracy) required for the reason of a trivial parallelization: $M=10^{5}$ independent Brownian particles (trajectories) were propagated in parallel for doing ensemble averaging. Sufficiently large $t_{\max }$ is required given very long transient regimes. For the studied problem, in the neglect of memory effects, it suffices to use $\Delta t=0.01$ in numerics 28] done here with the second-order stochastic Heun algorithm [55]. In the presence of memory effects, five times smaller $\Delta t=0.002$ was sufficient.

For doing Markovian embedding, one introduces a set of $N$ auxiliary variables $u_{i}$ such that the corresponding embedding dynamics in the hyperspace of dimension $D=$ $N+2$ reads [18, 38]

$$
\begin{aligned}
\dot{x}(t) & =v(t) \\
m^{*} \dot{v}(t) & =f(x, t)-\sum_{i=1}^{N} u_{i}(t)-\left(\eta_{0}+\eta_{\Sigma}\right) v(t) \\
& +\xi_{0}(t)+\sqrt{2 k_{B} T \eta_{\Sigma}} \zeta_{0}(t) \\
\dot{u}_{i}(t) & =-k_{i} v(t)-\nu_{i} u_{i}(t)+\sqrt{2 k_{B} T k_{i} \nu_{i}} \zeta_{i}(t),
\end{aligned}
$$

for $i=1, \ldots, N$, where $\eta_{\Sigma}=\sum_{i=1}^{N} \eta_{i}$. Furthermore, $\xi_{0}(t)$ and $\zeta_{i}(t), i=1 . . N$, are $N+1$ delta-correlated in time and mutually uncorrelated white Gaussian noise sources of zero-mean and unit intensity, $\left\langle\zeta_{i}(t) \zeta_{j}\left(t^{\prime}\right)\right\rangle=\delta_{i j} \delta\left(t-t^{\prime}\right)$, for $i, j=1 . . N,\left\langle\xi_{0}(t) \zeta_{i}\left(t^{\prime}\right)\right\rangle=0$. However, the noise $\zeta_{0}(t)$ is chosen as a weighted, normalized sum of other $\zeta_{i}(t)$ [18, 38],

$$
\zeta_{0}(t)=\sum_{i=1}^{N} \sqrt{\frac{\eta_{i}}{\eta_{\Sigma}}} \zeta_{i}(t) .
$$

The initial $u_{i}(0)$ are sampled as independent Gaussian variables with zero mean and correlations $\left\langle u_{i}(0) u_{j}(0)\right\rangle=$ $k_{B} T k_{i} \delta_{i j}[38$, 44, 52]. Initially, particles were always prepared with their velocities Maxwell-distributed at temperature $T$ and localized sharply at $x=0$, which corresponds to the minimum of potential in the unbiased case $f=0$. One assumes besides that $v=0$ for $t<t_{0}=0$ in Eq. (11). This is a non-equilibrium initial preparation.

\section{Appendix B: Estimation of physical parameters.}

Here, we address physical systems, where the studied effects can be revealed experimentally. The crucial issue here is a sufficiently small non-dimensional $\tilde{\gamma}_{0}$, which can 
be expressed as

$$
\tilde{\gamma}_{0}=6 \sqrt{\frac{3 \pi}{2} \frac{\rho_{f}}{2 \rho+\rho_{f}}} \frac{x_{0}}{R} \theta,
$$

where $\theta=\sqrt{\frac{R \rho_{f}}{U_{0}}} \mu=\sqrt{\frac{R}{U_{0} \rho_{f}}} \zeta$, with $\zeta=\rho_{f} \mu$ being the dynamic viscosity. The particles diameter should not be much larger than the potential period $L=2 \pi x_{0}$. For this reason, $x_{0} / R$ could hardly be much smaller than 0.1 . For example, for Brownian particles in optical vortices in Ref. [22], $x_{0}=52.5 \mathrm{~nm}$ and $R=740 \mathrm{~nm}$. This yields $x_{0} / R \approx 0.0709$. To arrive at smallest $\tilde{\gamma}_{0}$, the strategy is hence to minimize $\theta$. The fluid with lowest known dynamic and kinematic viscosity is liquid helium. At $T=4$ $\mathrm{K}$, its dynamic viscosity is $\zeta=3.3 \times 10^{-6} \mathrm{~Pa} \cdot \mathrm{s}$ [56]. With density $\rho_{f}=125 \mathrm{~kg} / \mathrm{m}^{3}$ this yields $\mu=2.64 \times 10^{-8} \mathrm{~m}^{2} / \mathrm{s}$. Let us take $U_{0}=100 k_{B} T \approx 5.52 \times 10^{-21} \mathrm{~J}$. Then, $\theta \approx 3.417$, and for $\rho=\rho_{f}$, we obtain $\tilde{\gamma}_{0} \approx 1.823$, which is a bit smaller than $\tilde{\gamma}_{0}=2$ used in this paper. The corresponding time units in our simulations would be $\tau_{0}=1.26 \times 10^{-5} \mathrm{~s}$ in physical units. Hence, the maximal time $t_{\max }=10^{6}$ in our simulations would correspond to about 12.6 seconds. This is interesting because the regimes of anomalous diffusion caused by hydrodynamic memory effects can reach the time scale of seconds. The values $\tilde{\gamma}_{0}$ ten times smaller can be achieved if to downscale $x_{0}$ and $R$ by a factor of hundred, i.e., for nanoparticles of $R \sim 7.4 \mathrm{~nm}$. The corresponding periodic nanostructures to create, e.g., electrostatic periodic potentials for charged Brownian particles can be produced by nanolithography [49], and nanosized hollow particles with appropriate low mass densities can also be tailored [57]. Furthermore, for more common fluids like diethyl ether at $T_{r}=298 \mathrm{~K}, \zeta=0.224 \times 10^{-3} \mathrm{~Pa} \cdot \mathrm{s}$ and $\rho=713.4 \mathrm{~kg} / \mathrm{m}^{3}$. For $U_{0}=100 k_{B} T_{r} \approx 4.114 \times 10^{-19} \mathrm{~J}$, $R=740 \mathrm{~nm}, x_{0}=52.5 \mathrm{~nm}$ we obtain $\theta \approx 11.247$, and for $\rho=\rho_{f}, \tilde{\gamma}_{0} \approx 6$. Next, a 9 -fold reduction of both $R$ and $x_{0}$ would yield $\tilde{\gamma}_{0} \approx 2$. Hence, the study of inertial effects in Brownian motion, including hydrodynamic memory effects, should be experimentally feasible. Some significant experimental work in this direction was already done for parabolic potentials optically created [15 17]. The case of periodic potentials is, however, more challenging.

\section{Appendix C: Kinetic heating and velocity distribution}

In this Appendix, we discuss kinetic heating of Brownian particles, velocity distribution $P(v, t)$ responsible for this kinetic heating, and their relation to enormous diffusion enhancement.

To begin with, in the scaling of this work, renormalized mass $m^{*}=1$ and $k_{B}=1$. Initially, particle velocities are Maxwell-distributed, $P(v, 0)=\exp \left[-v^{2} /(2 T)\right] / \sqrt{2 \pi T}$ with zero mean, $\langle v(0)\rangle=0$, and the variance $\left\langle\delta v^{2}(0)\right\rangle=$ $v_{T}^{2}=T$. Here and in the following, $\delta v(t)=v(t)-\langle v(t)\rangle$.
In the absence of periodic potential and for an arbitrary strong force $f, P(v, t)$ after a transient time $1 / \gamma_{0}$ is Maxwell-distributed around the mean value $\langle v\rangle_{\mathrm{st}}=$ $f / \gamma_{0}$. Doing the overdamped limit at strong friction $\gamma_{0} \gg 1$, one assumes that velocity distribution remains Maxwellian (shifted by $\langle v\rangle$ ) at the same temperature $T$, and excludes the velocity variable from the further consideration. This assumption becomes questionable in the case of nonlinear driven dynamics even for a sufficiently large but finite $\gamma_{0}$. At the first look, dynamics in considered tilted washboard potentials can become close to the overdamped case already for $\gamma_{0}>\gamma_{0}^{(c)}=1.193$, as the Risken's phase diagram might suggest, see the memoryless case in Fig. 1 of the main text. Indeed, following this diagram, the only critical force, which seems relevant above $\gamma_{0}^{(c)}$, is $f_{c}^{(1)}=1$. For $\gamma_{0}>\gamma_{0}^{(c)}$ and $f<f_{c}^{(1)}$ at $T=0$ any particle will be eventually trapped in a potential well, whereas at $f>f_{c}^{(1)}$ it will be running. For $\gamma_{0}<\gamma_{0}^{(c)}$ at $f<f_{c}^{(3)}$ and $T=0$ all particles are eventually trapped, whereas for $f>f_{c}^{(3)}$ the running solutions appear. Risken defined an effective potential for a particle using its total energy as a variable, see Ch. 11.6 in Ref. 7]. It displays bistability at $f_{c}^{(3)}<f<f_{c}^{(1)}$ (in our notations) and at some critical force $f_{c}^{(2)}$, both minima of that effective potential become equal. In Refs. [26, 27], a velocity pseudo-potential, $V(v)=-k_{B} T \ln [P(v)]$, was considered instead of the Risken's potential, where $P(v)$ is the velocity distribution. It turns out to be also bistable for sufficiently small friction. One minimum at $v_{1}=0$ corresponds to the trapped particles, with velocity being Maxwell-distributed around this minimum, and another minimum is located at $v_{2}=f / \gamma_{0}$, with velocity also Maxwell-distributed around $v_{2}$. Bi-parabolic velocity pseudo-potential with cusp at intersection of two parabolas provides a reasonable approximation to $V(v)$ 26, 27. This picture of velocity bistability remains valid, upon some modifications, also in the presence of hydrodynamic memory effects for $\gamma_{0}=0.1$ in Ref. [18].

However, this simple picture breaks down even in the absence of memory effects for sufficiently strong friction exceeding (about) $\gamma_{0}=0.25$; see Supplemental Material [41] of Ref. 18], which was also confirmed quite recently in Ref. [42]. The numerical simulations reveal that already for $\gamma_{0}=0.3$, the velocity distribution can be three-modal, see Fig. 7, (a) in the discussed Supplemental Material [41] of Ref. [18], and, especially, the panel (c) therein, for $\gamma_{0}=0.7$. The running state consists, in fact, of two velocity substates with $P(v)$ maxima at $v_{2}^{(1)}$ and $v_{2}^{(2)}$ such that $v_{2}^{(1)}<v_{2}=f / \gamma_{0}<v_{2}^{(2)}$. Moreover, $v_{2}$ corresponds to the minimum (!) and not maximum of $P(v)$, as bistable picture of $V(v)$ [26, 27], valid for sufficiently small $\gamma_{0}$ only [18], can misleadingly imply. For $f \geq f_{c}^{(1)}$, $P(v)$ becomes bimodal in such a memoryless case because of the minimum at $v_{1}=0$, which corresponds to the trapped particles, disappears - see the panel $(d)$ in the discussed figure. These earlier overlooked features are 

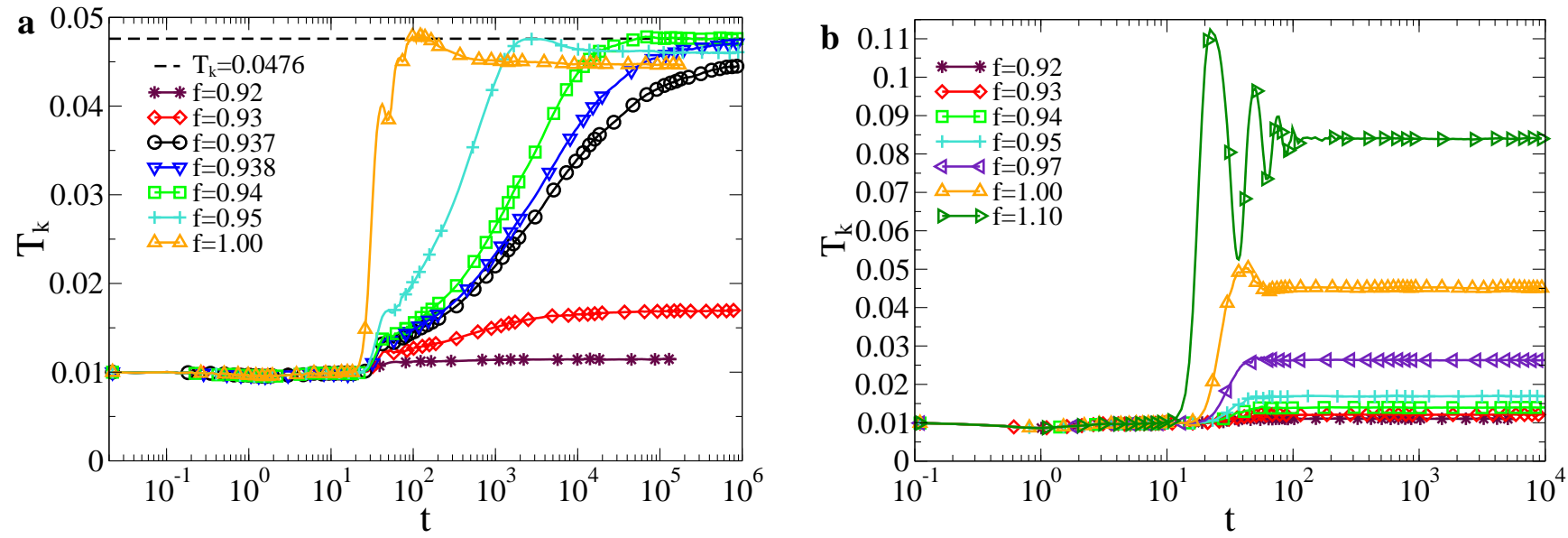

FIG. 6. Kinetic temperature defined as $T_{k}(t)=\left\langle\delta v^{2}(t)\right\rangle$ in the scaled units, vs. time at $T=0.01$ for $\gamma_{0}=2$, (a) $\gamma_{\alpha}=3$ $\left(\rho=\rho_{f} / 2\right)$ and (b) $\gamma_{\alpha}=0$ (normal diffusion) at several values of tilting force $f$ shown in plots. Initially, $T_{k}(0)=T$. The particles can first slightly cool down, when they start equilibrating being localized initially in a potential well. Then, they arrive again at $T$ during this equilibration process and start drastically heat up, when they leave the potential well, for a sufficiently large $f$ and $t$. In the case of normal diffusion, $T_{k}(t)$ arrives at a stationary non-equilibrium value $T_{k}^{(\text {st })}$ already for $t>100$. For $f<0.9$, the heating effect is almost negligible. However, the particles can become kinetically very hot even in the absence of memory effects. For example, at the critical tilt $f=1.00$ in panel (b) they are 4.5 times kinetically hotter than their surrounding. For strong memory effects in panel (a), the maximal $T_{k}^{\text {(st) }} \approx 4.76 T$ is arrived at $f=0.94$ in this panel. Then, with a further increasing $f, T_{k}^{(\mathrm{st})}$ gradually diminishes until it reaches $T$ for a very large $f$. However, in the memoryless case, $T_{\mathrm{h}}^{\text {(st) }}$ dramatically increases further with $f$ (see for $f=1.1$ ) until about $f=1.5$ (not shown) and only then gradually drops. $\mathrm{T}$
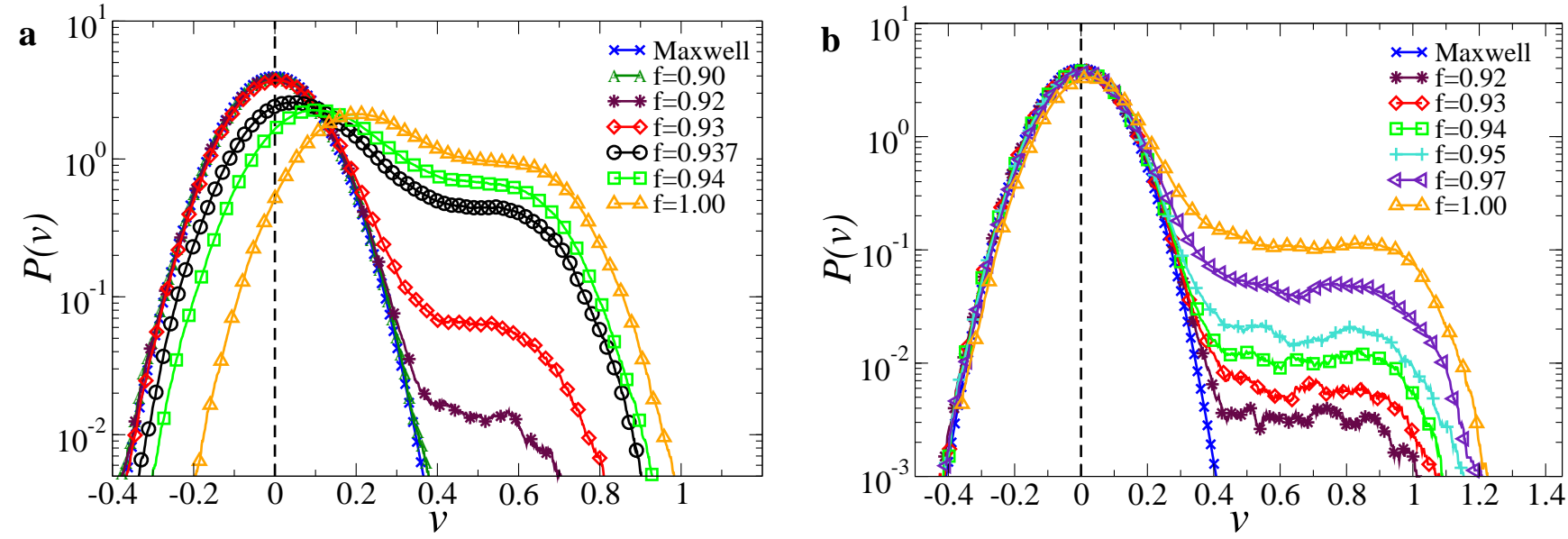

FIG. 7. Velocity distribution in case of (a) diffusion with memory and (b) memoryless diffusion at $T=0.01, \gamma_{0}=2$ and several force values shown in plots. In panel (a), $\gamma_{\alpha}=3$, which corresponds to $\rho=\rho_{f} / 2$. Maxwell equilibrium distribution is also shown for comparison. The emerging bimodality of $P(v)$ for a sufficiently large $f$ is obvious. For $f=0.937$ in panel (a) the distribution is still not stationary. $M=10^{5}$ particles are used to produce these distributions in each case considered.

important to understand the results of this work. The velocity distribution $P(v)$ can be bimodal even for not too large $\gamma_{0}$, well above the critical force $f_{c}^{(1)}$ (until some very large $f$ ), when the tilted washboard potential does not have anymore some minima and maxima at all. It is a great surprise overlooked until recently.

Generally, $P(v, t)$ is time-dependent for the considered nonlinear stochastic dynamics. For a sufficiently large time, a stationary distribution $P_{\mathrm{st}}(v)=\lim _{\rightarrow \infty} P(v, t)$ will be attained. However, this limit is not always possible to reach in our numerics, especially in the presence of memory effects. Although the memory effects do not affect $P_{\mathrm{st}}(v)$ in the case of linear dynamics, where it remains Maxwellian, they generally essentially influence both $P(v, t)$ and $P_{\mathrm{st}}(v)$ in the case of driven nonlinear dynamics considered.

The emerging very broad $P(v, t)$ velocity distribution, that is profoundly different from the Maxwell dis- 
tribution, means that particles become kinetically very hot. The kinetic temperature is commonly characterized by the velocity variance $\left\langle\delta v^{2}(t)\right\rangle$ such that $T_{k}(t)=$ $\left\langle\delta v^{2}(t)\right\rangle$ [26, 36 38]. For equilibrium Maxwell distribution, $T_{k}(t)=T$. It is so initially in Fig. 6. As everywhere else in this paper, the particles initially are localized at $x=0$, which is a nonequilibruim distribution within the potential well. During the initial equilibration they are first slightly cooled, and then heated up to $T$ again. Very interesting phenomenon occurs when the particles diffuse out of the initial potential well. Then, they can be heated up to some $T_{k}(t) \gg T$ reaching finally a stationary value $T_{k}^{(\mathrm{st})}$. If $f$ is far below $f_{c}^{(3)}, T_{k}^{(\mathrm{st})}=T$, as expected. For example, for $f=0.90$ in Fig. 7. (a), $P_{\text {st }}(v)$ is still practically Maxwellian, and no kinetic heating occurs. However, already for $f=f_{c}^{(3)}=0.92$ in panel (a) of Fig. 6. $T_{k}^{(\mathrm{st})} / T \approx 1.15$, i.e., $T_{k}$ is enhanced by about $15 \%$ over $T$. In the absence of memory effects, in panel (b), the enhancement is somewhat smaller: $10 \%$ only. The deviation from $T$ is still sufficiently small, in both cases. The onset of $P_{\mathrm{st}}(v)$ bistability is clearly seen in Fig. 7 for $f=0.92$, also in the absence of memory effects, cf. panel (b) therein. However, already for $f=0.93$, the kinetic temperature increases by ca. $70 \%$ in panel (a), Fig. 6. vs. $21 \%$ in panel (b). Astoundingly, a tiny further increase of force to $f=0.94$ boosts $T_{k}^{(\mathrm{st})}$ to about $T_{k}^{(\mathrm{st})}=4.76 \mathrm{~T}$ in panel (a), which is the maximal stationary value therein. This sharp increase should be contrasted with a still small increase in the memoryless case in Fig. 6. (b). Hence, the discussed sharp increase is caused by the memory effects indeed. With a further increase of $f, T_{k}^{(\mathrm{st})}$ gradually diminishes and for a very large force it finally drops down to $T$ again (not shown). The force range, where the Brownian particles become hot under constant driving is surprisingly large. It must be mentioned also that for $f=f_{c}^{(2)}=0.937$ and $f=0.938, T_{k}(t)$ still did not reach the stationary value $T_{k}^{(\mathrm{st})}$ in panel (a) of Fig. 6] The corresponding distribution $P(v)$ for $f=0.937$ in Fig. 7. (a) is still not stationary. This still increasing $T_{k}(t)$ in Fig. 6 correlates with transient superdiffusion which lasts until the end of simulations in these cases, see Fig. 3, (a) of the main text. Maximum of the corresponding asymptotic diffusion coefficient estimated in Fig. 4, (a) of the main text indeed seems to be associated with the maximum of $T_{k}^{(\mathrm{st})}$ vs. $f$, as in the case of small $\gamma_{0}$ [18]. However, here some profound warnings are due. First, even if $T_{k}(t)$ is already saturated for $f=0.94$ in the discussed case, cf. panel (a) of Fig. 6] the corresponding diffusional behavior in Fig. 3, (a) of the main text is still transient, and, unexpectedly, displays subdiffusion. For a much smaller $\gamma_{0}=0.1$ in Ref. [18] the emergence of such a transient subdiffusion regime was connected with the regime like one for $f=0.95$ in panel (a) of Fig. 6. when $T_{k}(t)$ drops gradually to $T_{k}^{(\mathrm{st})}$ after reaching a maximum. Indeed, also for this case, a transient subdiffusive behavior is still detectable (not shown). However, already for $f=1$, one cannot find such a regime, even if the corresponding non-monotonous behavior of $T_{k}(t)$ in panel (a) of Fig. 6 might imply it. It becomes simply too short to be detectable. Second, in the memoryless case, the maximum of $T_{k}^{(\mathrm{st})}$ does not correspond to the maximum of $D$. Indeed, in Fig. 6 (b), $T_{k}^{(\mathrm{st})}$ is the largest for $f=1.1$ and not for $f=1.0$ which corresponds to the maximum of $D$ in Fig. 4, (a) of the main text. In other words, increase of $f$ beyond $f_{c}^{(1)}$ in the memoryless case leads to a further increase of $T_{k}^{(\mathrm{st})}$, cf. Fig. 6. (b), whereas $D$ already starts to diminish, cf. Fig. 4, (a) of the main text. Here, a fascinating novel phenomenon emerges, which seems to be completely overlooked thus far and which we defer to a separate study. Namely, the stationary velocity autocorrelation function (VACF) starts, to our great surprise, rapidly oscillate in time (not shown). These oscillations correlate with transient $T_{k}(t)$ oscillations like ones for $f=1.1$ in Fig. 6 (b), which are also quite surprising. Since the diffusion coefficient is integral of VACF [11, 45], $D$ declines with $f$ despite $T_{k}^{(\mathrm{st})}$ still grows. This doubly unusual phenomenon indicating that the inertial effects can remain significant for appreciably large $\gamma_{0}$, even in the absence of hydrodynamic memory effects, will be studied in a separate work. It is especially striking and surprising because the diffusion enhancement in this work is already well described by the results of overdamped theory in the corresponding case.

The behavior of $P(v)$ in Fig. 7 also deserves a separate discussion. Notice that at $f=0.90$ in panel (a), $P(v)$ is still pretty well described by the equilibrium Maxwell distribution, with the center which is still practically not shifted. For $f=0.92$, the emerging velocity bistability becomes perspicuous. However, the first maximum is still centered at $v=0$ corresponding to the trapped states. Nevertheless, already for $f=0.93$, this maximum shifts slightly to the running states, indicating that for a finite temperature, the trapped states are destabilized by thermal fluctuations for $f>f_{c}^{(3)}$, in the case considered, which is a remarkable feature. Likewise, already at $f=1$ in the case of memoryless diffusion, the first maximum of $P(v)$ shifts to the running states, cf. Fig. 7 (b). One might expect it because, at $f=1$, the potential minima vanish overall. However, such a distinct shift at $f=0.937$ in panel (a) is quite surprising.
[1] J. Boussinesq, Sur la resistance qu'oppose un fluide indefini au repos, C.R. Acad. Paris 100, 935 (1885).
[2] A. B. Basset, A Treatise on Hydrodynamics, Vol. 2 (Deighton Bell, Cambridge, 1888) pp. 285-297. 
[3] L. D. Landau and E. M. Lifshitz, Fluid Mechanics, 2nd ed. (Pergamon Press, Oxford, 1987).

[4] O. Druzhinin and L. Ostrovsky, The influence of Basset force on particle dynamics in two-dimensional flows, Physica D 76, 34 (1994).

[5] K. Guseva, U. Feudel, and T. Tél, Influence of the history force on inertial particle advection: Gravitational effects and horizontal diffusion, Phys. Rev. E 88, 042909 (2013).

[6] E. Frey and K. Kroy, Brownian motion: a paradigm of soft matter and biological physics, Annalen der Physik 14, 20 (2005).

[7] H. Risken, The Fokker-Planck Equation: Methods of Solution and Applications, 2nd ed. (Springer, Berlin, 1989).

[8] W. T. Coffey and Y. P. Kalmykov, The Langevin Equation: With Applications to Physics, Chemistry and Electrical Engineering, 3rd ed. (World Scientific, Singapore, 2012).

[9] P. Hänggi, P. Talkner, and M. Borkovec, Reaction-rate theory: fifty years after Kramers, Rev. Mod. Phys. 62, 251 (1990)

[10] A. Widom, Velocity Fluctuations of a Hard-Core Brownian Particle, Phys. Rev. A 3, 1394 (1971).

[11] R. Kubo, M. Toda, and M. Hashitsume, Statistical Physics II, Nonequilibrium Statistical Mechanics (Springer, Berlin, 1985).

[12] B. J. Alder and T. E. Wainwright, Velocity Autocorrelations for Hard Spheres, Phys. Rev. Lett. 18, 988 (1967).

[13] F. Mainardi and P. Pironi, The Fractional Langevin Equation: Brownian Motion Revisited, Extr. Math. 10, 140 (1996).

[14] D. A. Weitz, D. J. Pine, P. N. Pusey, and R. J. A. Tough, Nondiffusive Brownian motion studied by diffusing-wave spectroscopy, Phys. Rev. Lett. 63, 1747 (1989).

[15] T. Franosch, M. Grimm, M. Belushkin, F. M. Mor, G. Foffi, L. Forro, and S. Jeney, Resonances arising from hydrodynamic memory in Brownian motion, Nature (London) 478, 85 (2011).

[16] R. Huang, I. Chavez, K. M. Taute, B. Lukic, S. Jeney, M. G. Raizen, and E.-L. Florin, Direct observation of the full transition from ballistic to diffusive Brownian motion in a liquid, Nat. Phys. 7, 576 (2011).

[17] S. Kheifets, A. Simha, K. Melin, T. Li, and M. G. Raizen, Observation of Brownian Motion in Liquids at Short Times: Instantaneous Velocity and Memory Loss, Science 343, 1493 (2014).

[18] I. Goychuk, Fractional Hydrodynamic Memory and Superdiffusion in Tilted Washboard Potentials, Phys. Rev. Lett. 123, 180603 (2019).

[19] G. Costantini and F. Marchesoni, Threshold diffusion in a tilted washboard potential, EPL 48, 491 (1999)

[20] P. Reimann, C. Van den Broeck, H. Linke, P. Hänggi, J. M. Rubi, and A. Pérez-Madrid, Giant Acceleration of Free Diffusion by Use of Tilted Periodic Potentials, Phys. Rev. Lett. 87, 010602 (2001).

[21] B. Lindner, M. Kostur, and L. Schimansky-Geier, Optimal diffusive transport in a tilted periodic potential, Fluct. Noise Lett. 01, R25 (2001).

[22] S.-H. Lee and D. G. Grier, Giant Colloidal Diffusivity on Corrugated Optical Vortices, Phys. Rev. Lett. 96, 190601 (2006).

[23] M. Evstigneev, O. Zvyagolskaya, S. Bleil, R. Eichhorn, C. Bechinger, and P. Reimann, Diffusion of colloidal particles in a tilted periodic potential: Theory versus ex- periment, Phys. Rev. E 77, 041107 (2008)

[24] J. M. Sancho, A. M. Lacasta, K. Lindenberg, I. M. Sokolov, and A. H. Romero, Diffusion on a Solid Surface: Anomalous is Normal, Phys. Rev. Lett. 92, 250601 (2004).

[25] K. Lindenberg, J. M. Sancho, A. M. Lacasta, and I. M. Sokolov, Dispersionless Transport in a Washboard Potential, Phys. Rev. Lett. 98, 020602 (2007).

[26] I. G. Marchenko and I. I. Marchenko, Diffusion in the systems with low dissipation: Exponential growth with temperature drop, EPL 100, 50005 (2012).

[27] I. G. Marchenko, I. I. Marchenko, and A. V. Zhiglo, Particle transport in space-periodic potentials in underdamped systems, Eur. Phys. J. B 87, 10 (2014).

[28] B. Lindner and I. M. Sokolov, Giant diffusion of underdamped particles in a biased periodic potential, Phys. Rev. E 93, 042106 (2016).

[29] H. D. Vollmer and H. Risken, Bistability effects of the brownian motion in periodic potentials, Z. Phys. B 37, 343 (1980).

[30] F. Marchesoni, Comment on stochastic resonance in washboard potentials, Phys. Lett. A 231, 61 (1997).

[31] I. G. Marchenko, I. I. Marchenko, and V. I. Tkachenko, Temperature-Abnormal Diffusivity in underdamped spatially periodic systems, JETP Lett. 106, 242 (2017).

[32] T. Geisel, A. Zacherl, and G. Radons, Generic $\frac{1}{f}$ Noise in Chaotic Hamiltonian Dynamics, Phys. Rev. Lett. 59, 2503 (1987).

[33] G. Zumofen and J. Klafter, Scale-invariant motion in intermittent chaotic systems, Phys. Rev. E 47, 851 (1993).

[34] M. F. Shlesinger, G. M. Zaslavsky, and J. Klafter, Strange kinetics, Nature 363, 31 (1993).

[35] B. Lindner and E. M. Nicola, Critical Asymmetry for Giant Diffusion of Active Brownian Particles, Phys. Rev. Lett. 101, 190603 (2008)

[36] N. V. Brilliantov and T. Pöschel, Kinetic Theory of Granular Gases (Oxford University Press, Oxford, 2004).

[37] P. Siegle, I. Goychuk, and P. Hänggi, Origin of Hyperdiffusion in Generalized Brownian Motion, Phys. Rev. Lett. 105, 100602 (2010).

[38] P. Siegle, I. Goychuk, and P. Hänggi, Markovian embedding of fractional superdiffusion, EPL 93, 20002 (2011).

[39] P. Siegle, I. Goychuk, P. Talkner, and P. Hänggi, Markovian embedding of non-Markovian superdiffusion, Phys. Rev. E 81, 011136 (2010).

[40] S. L. Seyler and S. Pressé, Long-time persistence of hydrodynamic memory boosts microparticle transport, Phys. Rev. Research 1, 032003 (2019).

[41] Supplemental Material of Ref. [18] at http:// link.aps.org/supplemental/10.1103/PhysRevLett.123.180603.

[42] J. Spiechowicz and J. Łuczka, Diffusion in a biased washboard potential revisited, Phys. Rev. E 101, 032123 (2020).

[43] E. Lutz, Fractional Langevin equation, Phys. Rev. E 64, 051106 (2001).

[44] I. Goychuk, Viscoelastic Subdiffusion: Generalized Langevin Equation Approach, Adv. Chem. Phys. 50, 187 (2012).

[45] P. M. Chaikin and T. C. Lubensky, Principles of Condensed Matter Physics (Cambridge University Press, Cambridge, 1995) pp. 413-414.

[46] A. M. Mathai and H. J. Haubold, An Introduction to Fractional Calculus (Nova Science Publishers, New York, 2017). 
[47] R. Kubo, Fluctuation-Dissipation Theorem, Rep. Prog. Theor. Phys. 29, 255 (1966).

[48] B. Mandelbrot and J. van Ness, Fractional Brownian motion, fractional Gaussian noise and applications, SIAM Rev. 10, 422 (1968).

[49] L. J. Guo, Nanoimprint lithography: methods and material requirements, Adv. Materials 19, 495 (2007).

[50] S. Lifson and J. L. Jackson, On the self-diffusion of ions in a polyelectrolyte solution, J. Chem. Phys. 36, 2410 (1962).

[51] J. Spiechowicz, J. Luczka, and P. Hänggi, Transient anomalous diffusion in periodic systems: ergodicity, symmetery breaking and velocity relaxation, Sci. Rep. 6, 30948 (2016).
[52] I. Goychuk, Viscoelastic subdiffusion: from anomalous to normal, Phys. Rev. E 80, 046125 (2009).

[53] B. D. Hughes, Random Walks and Random Environments (Clarendon Press, Oxford, 1995).

[54] R. G. Palmer, D. L. Stein, E. Abrahams, and P. W. Anderson, Models of Hierarchically Constrained Dynamics for Glassy Relaxation, Phys. Rev. Lett. 53, 958 (1984).

[55] T. C. Gard, Introduction to Stochastic Differential Equations (Dekker, New York, 1988).

[56] B. Hands, Cryogenic engineering (Academic Press, London, 1986).

[57] K. C. Bentz and D. A. Savin, Hollow polymer nanocapsules: synthesis, properties, and applications, Polym. Chem. 9, 2059 (2018). 